\title{
Fan Noise Prediction and Control Research of Turbofan Engine
}

\author{
Wenliang Zhang ${ }^{1, a, *}$, Jun Huang ${ }^{1, b}$, Mingxu Yi ${ }^{1, c}$, Yacong Wu ${ }^{1, d}$ \\ ${ }^{1}$ School of Aeronautic Science and Engineering, Beihang University, College Road No.37, Beijing, \\ China \\ a'zwlsygxsg@163.com, buunhuang1964@163.com, ${ }^{\mathrm{a} y i m i n g x u 88 @ 163 . c o m, ~}{ }^{\mathrm{d}}$ wycbuaa@163.com
}

Keywords: fan noise, noise control, turbofan engine fan

\begin{abstract}
This research aims to study the acoustic noise prediction and control of turbofan engine fan. In this paper, a thin-body BEM/FW-H method is developed to efficiently predict the propagation of fan sound wave in the duct. It is a hybrid method combining computational aeroacoustics with Boundary Element Method (BEM). And two main geometry parameters are modified to investigate the noise reduction of turbofan engine fan by using the numerical prediction method. The acoustic performance of different modification tasks is discussed. These results verify the efficiency of design parameters modification to reduce acoustic noise of turbofan engine fan.
\end{abstract}

\section{Introduction}

Aircraft noise problems raised an important question for the air transport industry with sustained growth. The obtaining of the aircraft airworthiness certification directly depends on its noise level. It is undoubtedly a huge challenge to the developing of China's civil aircraft. Fan noise is the primary sound source of modern civil aircraft during takeoff and landing condition. As the engine noise is caused largely due to the fan, and fan noise is proportional to the 4th power of the blade tip tangential velocity. As for acoustic noise of fan and duct, previous researches on the generation and propagation of the noise of rotor and axial fan have yielded large amount of good results. Generally, the noise source is expressed using acoustic analogy and the flow field is solved using Computational Fluid Dynamics (CFD) or measured by experimental means [1,2]. Over the last two decades, the Boundary Element Method (BEM) has become a valuable modeling especially for problems in acoustics and noise control.Improved understanding of the acoustic noise reduction can lead to quieter engine designs. Some researchers have studied the acoustic noise control of turbofan engine. Tyler and sofrin. [3] carried out the pioneer work based on the acoustic mode, describing the sound field in the engine pipeline, and proposed the mechanism of the noise-mode of the rotor-stator interaction at the subsonic tip speed. Cao et al. [4] studied the influence of speed, number of blades, wheel ratio, blade mounting angle and blade shape on the aerodynamic noise of the fan, and the optimal value of each parameter was selected to optimize the design and noise reduction. Akaike et al. [5] studied 
non-equidistant blade distribution for noise reduction, numerical and experimental results show that the fan's rotation noise to a certain extent.

This paper focuses on a discussion of turbofan engine fan modification based on noise reduction. The hybrid method combining thin-body BEM and FW-H method is developed to predict the acoustic noise of turbofan engine fan in this research. Then two main geometry parameters are modified to reduce the noise of turbofan engine fan. The acoustic performance of different modification tasks is discussed and shown in this research.

\section{Numerical methods}

The noise of turbofan engine fan is generated from the pressure fluctuation of the blade surface, which is caused by the unsteady aerodynamic forces. The noise of fan is composed of incident sound generated by blade and scattered sound generated by duct. The thin-body BEM/FW-H numerical method is divided into three steps: firstly, the sound source generated from the pressure fluctuations of the blade surface is calculated using CFD method; secondly, the free-field sound pressure is calculated using FW-H method, and then it's transformed into sound pressure in frequency domain using Fast Fourier Transform (FFT) method; lastly, the scattering effect of the duct wall is solved using thin-body BEM method, and the radiation and propagation of the sound source is calculated.

\subsection{Unsteady flow calculation}

Accurate calculation of the flow field is very essential for the calculation of acoustic noise. Due to the constant rotary speed, we focus on the flow field in stable state. Therefore, the unsteady Navier-Stokes equation is adopted here to calculate the flow field [6].

In this research, the flow field properties are calculated by using the commercial CFD code FLUENT 14.0, which has a lot of turbulence models such as model, SST model and LES model. The standard model is used in this research for its reasonable solving accuracy and fewer resources compared with SST model and LES model.

The no-slip wall is adopted in this aerodynamic simulation. The SIMPLEC discretization schemes are used to discretize the equation of pressure-velocity coupling. The rotating speed of turbofan engine fan modification is $4000 \mathrm{r} / \mathrm{min}$. And the time step in relation to the rotating speed is given in order to obtain enough calculated results per blade passing time.

\subsection{Free field acoustic source}

Acoustic analogy can be used to study the problem of aerodynamically generated noise, and the FW-H equation is adopted to calculate the free-field acoustic noise [7, 8]. FW-H equation can be solved by using the formulation of Farassat $1 \mathrm{~A}$, which is given as follows:

$$
p^{\prime}(x, t)=p_{T}{ }^{\prime}(x, t)+p_{L}{ }^{\prime}(x, t)
$$

with

$$
4 \pi p_{T}{ }^{\prime}(x, t)=\int_{S}\left[\frac{\rho_{0}\left(\dot{U}_{n}+U_{\dot{n}}\right)}{r\left(1-M_{r}\right)^{2}}\right]_{r e t} d S+\int_{S}\left[\frac{\rho_{0} U_{n}\left(r_{i} \dot{M}_{i}+c_{0} M_{r}-c_{0} M^{2}\right)}{r^{2}\left(1-M_{r}\right)^{3}}\right]_{r e t} d S
$$




$$
\begin{aligned}
4 \pi p_{L}{ }^{\prime}(x, t) & =\frac{1}{c_{0}} \int_{S}\left[\frac{\dot{L}_{r}}{r\left(1-M_{r}\right)^{2}}\right]_{r e t} d S+\int_{S}\left[\frac{L_{r}-L_{M}}{r^{2}\left(1-M_{r}\right)^{2}}\right]_{r e t} d S \\
& +\frac{1}{c_{0}} \int_{S}\left[\frac{L_{r}\left(r \dot{M}_{r}+c_{0} M_{r}-c_{0} M^{2}\right)}{r^{2}\left(1-M_{r}\right)^{3}}\right]_{r e t} d S
\end{aligned}
$$

where $p_{T}{ }^{\prime}(x, t)$ represents thickness sound pressure and $p_{L}{ }^{\prime}(x, t)$ represents loading sound pressure. $\rho_{0}$ and $c_{0}$ are the fluid and sound speed, respectively. $L$ represents the aerodynamic pressure with $L_{i}=P_{i j} \hat{n}_{j}+\rho u_{i}\left(u_{n}-v_{n}\right) \cdot u_{i}$ is the fluid velocity and $v_{i}$ represents the velocity of the surface. The relative speed $\left(u_{i}-v_{i}\right)=0$ and $U_{i}$ is reduced to $U_{i}=v_{i}$ when the control surface is solid. $\boldsymbol{M}$ represents local Mach number vector of source with respect to a frame fixed to the undisturbed medium, with components $M_{i}$. And the subscript $r$ denotes projection onto the source observer direction. The subscript $M$ denotes projection on the Mach number vector.

After the free-field sound pressure is calculated using FW-H method, it is transformed into sound pressure in frequency domain by FFT method.

\subsection{Acoustic thin-body BEM for sound propagation}

The BEM method is used to calculate the far-field sound pressure. Due to the scattering effect of the duct wall, the total sound pressure is the superposition of incident pressure and scattered pressure $[9,10]$. The incident pressure in frequency domain can be solved using the method mentioned in previous sections, and the scattered pressure can be solved using BEM.

$$
p_{\text {sum }}^{\prime}=p_{i}^{\prime}+p_{s}^{\prime}
$$

where $p_{i}^{\prime}$ and $p_{s}^{\prime}$ separately denote incident sound pressure and scattered sound pressure in the frequency domain. In this research, the scattered sound pressure is calculated using the thin-body boundary integral equation. The calculated domain is shown in Fig. 1, the surface of the duct wall is expressed by $S$. Since the duct is not close, an imaginary surface $S_{i}$ is constructed to divide the acoustic domain into an interior domain $V^{-}$and an exterior domain $V^{+}$. The sound pressure on the inside of the closed surface is denoted as $p^{-}$while outside is $p^{+}$. The integral equation is

$$
\begin{array}{r}
\int_{S}\left(p^{+}-p^{-}\right) \frac{\partial \psi}{\partial n} d S=\frac{1}{2}\left[p^{+}(x)-p^{-}(x)\right] \quad x \in S \\
\int_{S}\left(p^{+}-p^{-}\right) \frac{\partial \psi}{\partial n} d S=p(x) \quad x \in V^{-} \cup V^{+}
\end{array}
$$




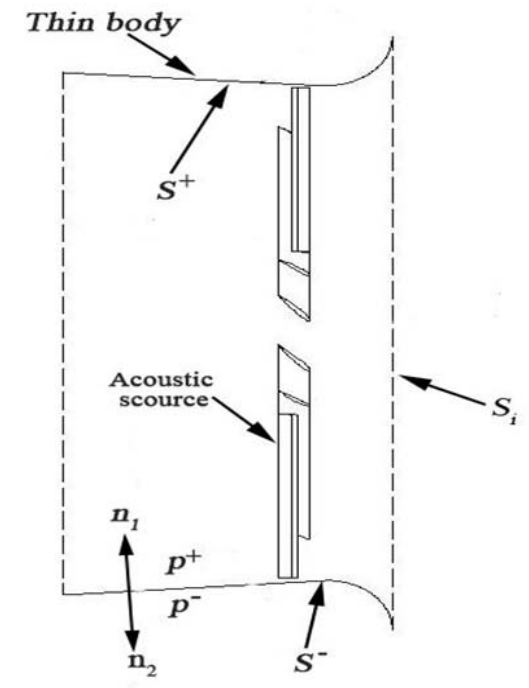

Fig. 1 The schematic diagram of acoustic scattering by a thin body

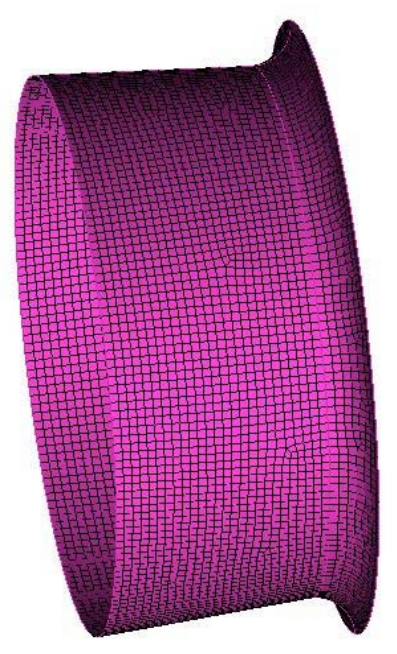

Fig. 2 Mesh of duct used in BEM

In order to calculate the scattered sound pressure, the thin-body neutral surface $S$ is discretized into a number of elements. Using the Linear BEM, the sound pressure jump $\left(p^{+}-p^{-}\right)$can be calculated by solving a set of equations. Then the scattered sound pressure at any elements can be solved directly by Eq. (5) and Eq. (6). The mesh of duct surface used in BEM is shown as Fig. 2.

An effort to realize the numerical noise prediction of turbofan engine fan was made through solving Eq. (4).

\section{Model establishment}

\subsection{Geometric configuration and grid generation}

A turbofan engine fan system is designed and tested to analyze and reduce its acoustic noise in this part. The virtual prototype model of turbofan engine fan is set up in Tab. 1, and its geometric model is shown in Fig. 3.

Table 1 Parameters of benchmark model of turbofan engine fan

\begin{tabular}{|c|c|}
\hline Structural parameter & Size \\
\hline Rotor radius R & $500 \mathrm{~mm}$ \\
\hline Blade aspect ratio & 3 \\
\hline Blade angle $\phi$ & $64^{\circ}$ \\
\hline Number of rotor blades $B$ & 17 \\
\hline Wheel ratio & 0.3 \\
\hline Duct Maximum cross section radius & $540 \mathrm{~mm}$ \\
\hline Rotor disc position $(0.282 \mathrm{~L})$ & $628 \mathrm{~mm}$ \\
\hline Length of duct $L$ & $2227 \mathrm{~mm}$ \\
\hline
\end{tabular}



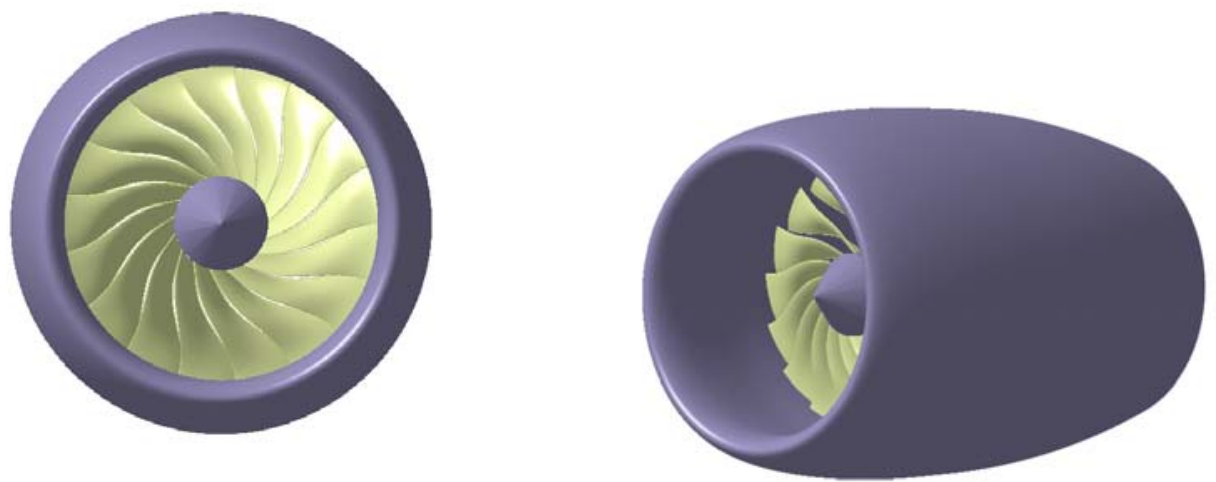

Fig. 3 Geometric model of turbofan engine fan

In the aerodynamics simulation of turbofan engine fan, the whole computation domain is divided into two subdomains: an independent rotational domain for the rotor disk and an outer stationary domain. The stationary domain includes the part of duct and rotor hub. After the geometry of turbofan engine fan is established, the calculation model is meshed by the unstructured triangle mesh, and the grid density can be controlled to improve computational precision. The grids in the rotational domain and duct domain are dense, as shown in Fig. 4. In consideration of the inlet and outlet zone (shown in Fig. 5), the total number of cells in the whole flow field is about 2.2 million.

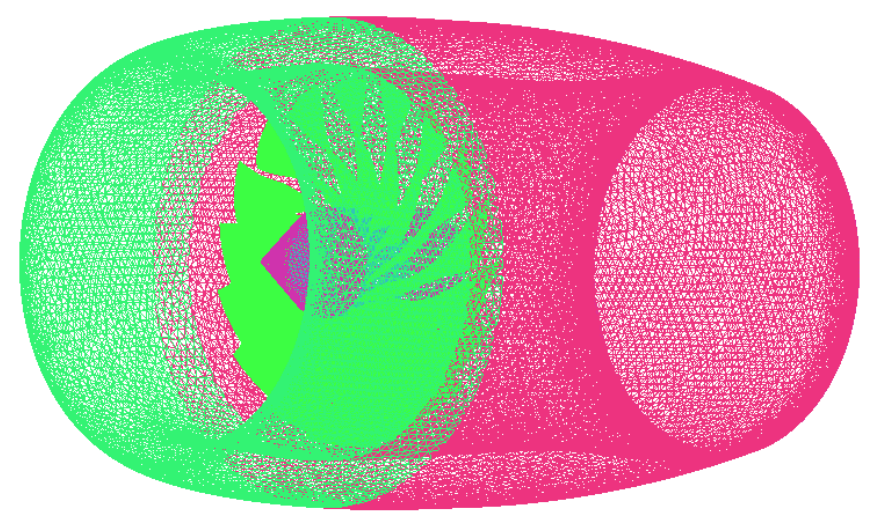

Fig. 4 Gird of turbofan engine fan

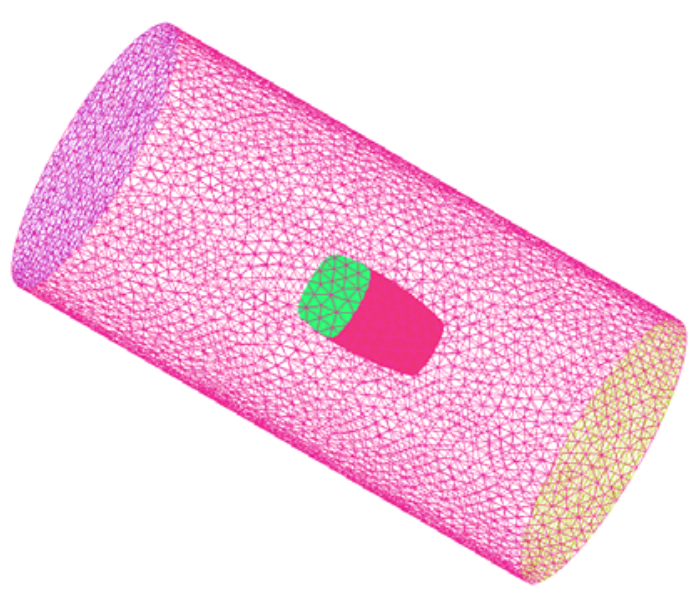

Fig. 5 Gird of the far-field

\subsection{Acoustic noise of turbofan engine fan}

In this simulation, all of the acoustic noise and SPLs of turbofan engine fan prototype model and the following different modifications are calculated at rotary speed of 4000rpm at the observation distance of $3 \mathrm{~m}$.

The sound pressure is expressed in decibels $(\mathrm{dB})$, and SPL is calculated by the following equation:

$$
S P L=P_{e} / P_{\text {ref }}
$$

where $P_{e}$ denotes the predicted pressure, $P_{\text {ref }}$ denotes the reference pressure and equals to $2 \times 10^{-5} \mathrm{~Pa}$. 


\section{Applications in acoustic noise reduction}

In this part, the numerical prediction method mentioned above is applied to study the acoustic noise reduction of turbofan engine fan. By analyzing the amendable geometrical parameters that have an obvious influence on acoustic noise, two design parameters including blade mounting angle and wheel ratio are altered to analyze the acoustic characteristics of turbofan engine fan.

The influence on acoustic noise of blade airfoil was considered in the first place. Four blade mounting angle were selected, i.e. $61^{\circ}, 64^{\circ}, 67^{\circ}$ and $70^{\circ}$. The blade mounting angle of original model is $64^{\circ}$.Then, the influence on acoustic noise from wheel ratio which was modified from 0.25-0.4 was discussed, as shown in Tab. 3. The SPLs turbofan engine fan calculated for various blade mounting angle and wheel ratio are shown as Fig.6 and Fig.7.

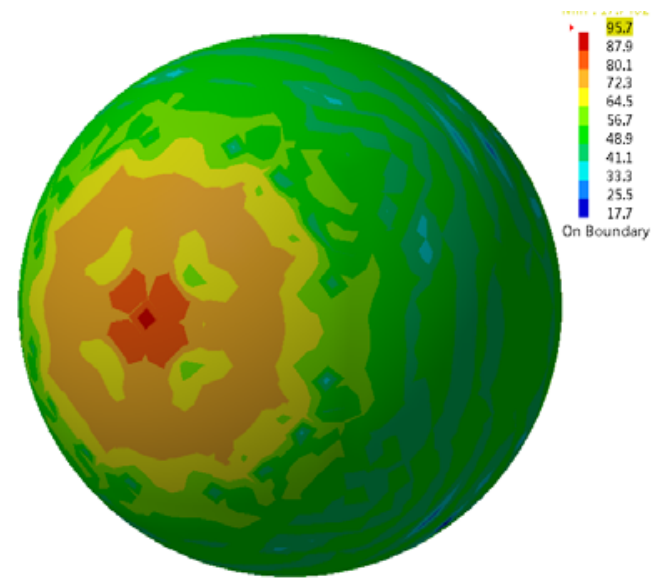

(a) SPLs of blade mounting angle $61^{\circ}$

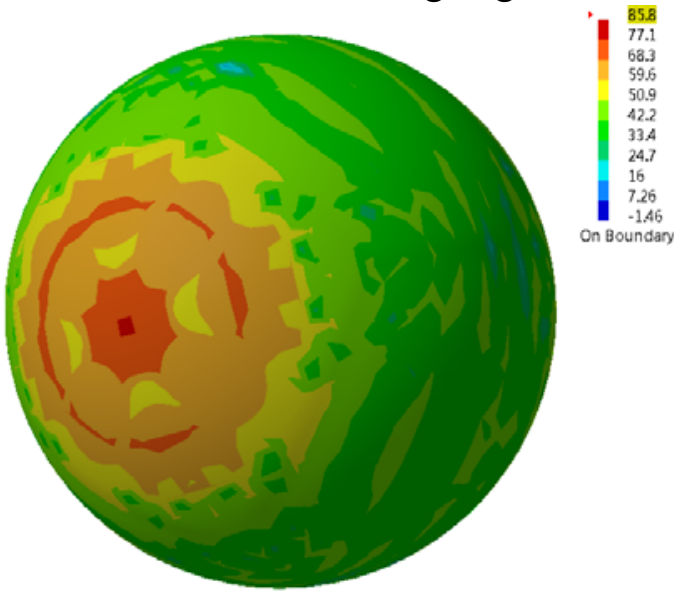

(c) SPLs of blade mounting angle $67^{\circ}$

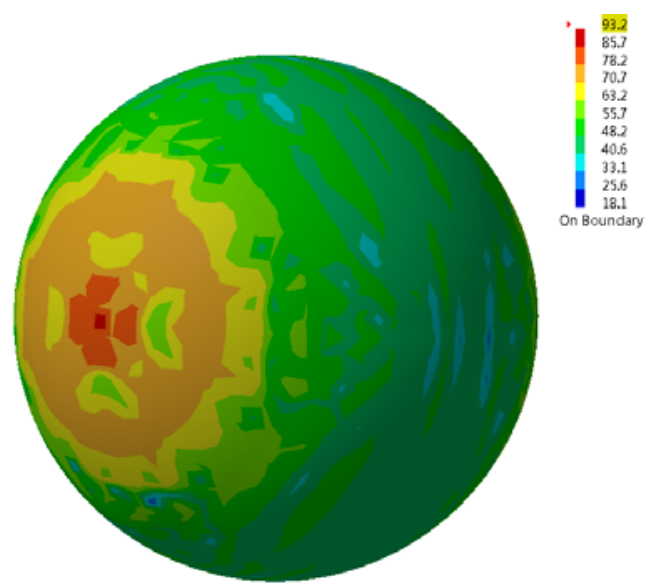

(b) SPLs of blade mounting angle $64^{\circ}$

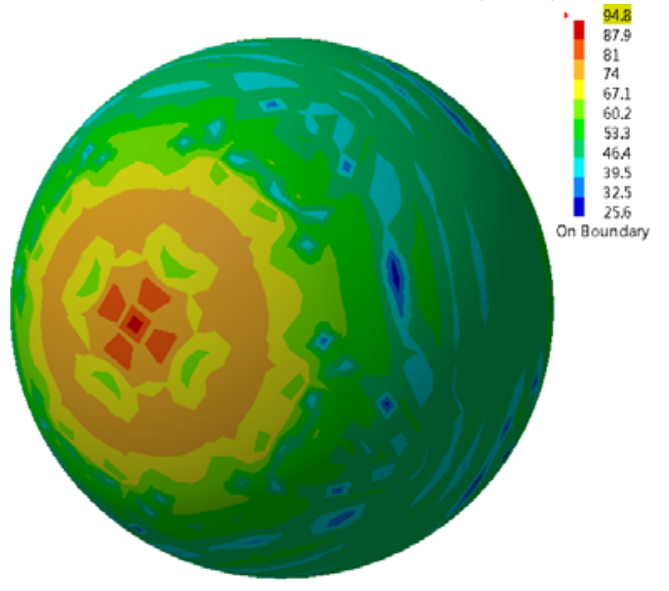

(d) SPLs of blade mounting angle $70^{\circ}$

Fig. 6 SPLs of different blade mounting angle modification tasks 
Table 2 Modification tasks of blade mounting angle and corresponding maximum SPLs

\begin{tabular}{|l|c|c|c|c|}
\hline Task & 1 & 2 & 3 & 4 \\
\hline Angle $\left({ }^{\circ}\right)$ & 61 & 64 & 67 & 70 \\
\hline Thrust $(\mathrm{N})$ & 2088.23 & 2643.08 & 3110.42 & 3538.15 \\
\hline SPLs $(\mathrm{dB})$ & 95.7 & 93.2 & 85.8 & 94.8 \\
\hline
\end{tabular}

These four blade mounting angle have different thrust and the thrust generated by the fan increases as the blade mounting angle increases. However the maximum SPLs of blade mounting angle $61^{\circ}$, $64^{\circ}$ and $70^{\circ}$ are higher (Tab. 2; Fig. 6). On the whole, the lowest noise blade mounting angle is $67^{\circ}$, whose maximum SPL is $85.8 \mathrm{~dB}$ (Tab. 2; Fig. 6).

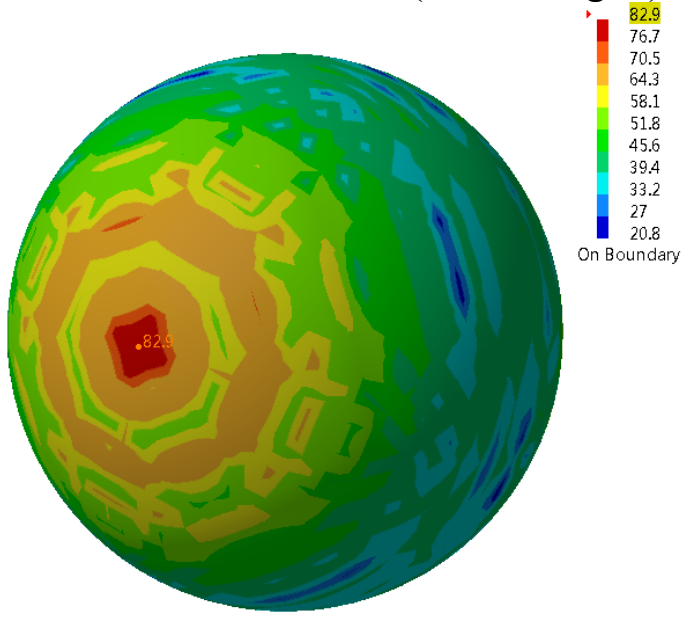

(a) SPLs of wheel ratio 0.25

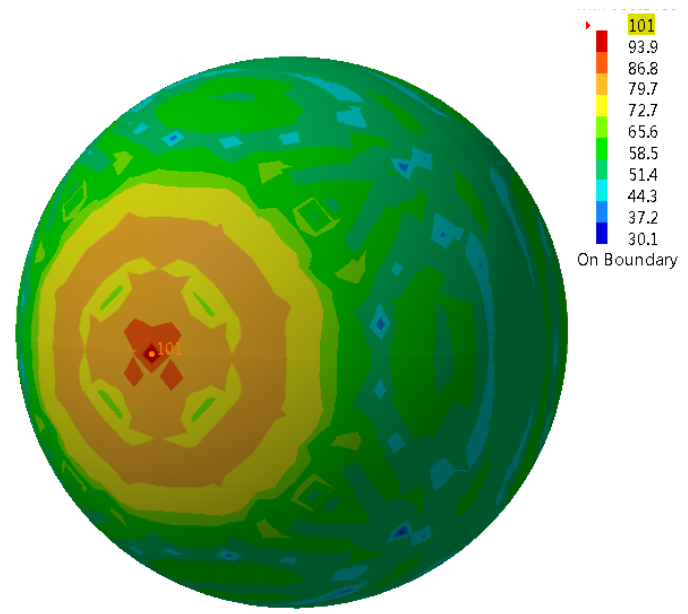

(c) SPLs of wheel ratio 0.35

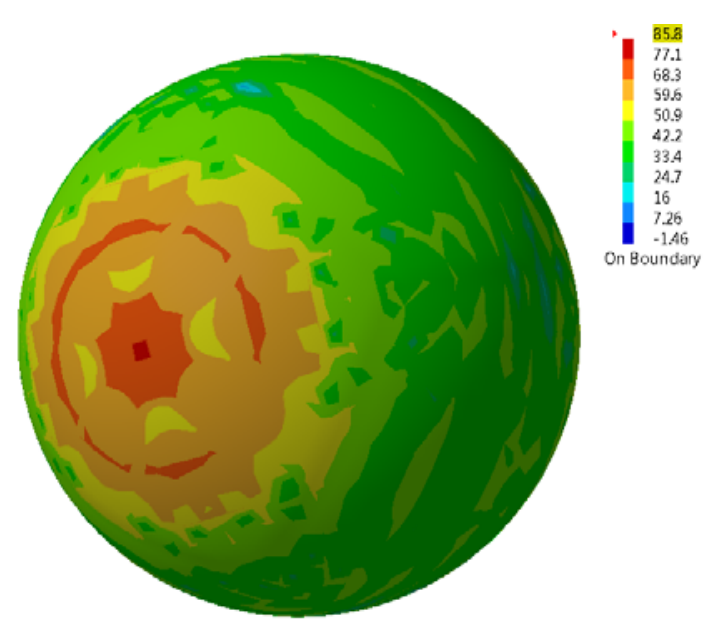

(b) SPLs of wheel ratio 0.3

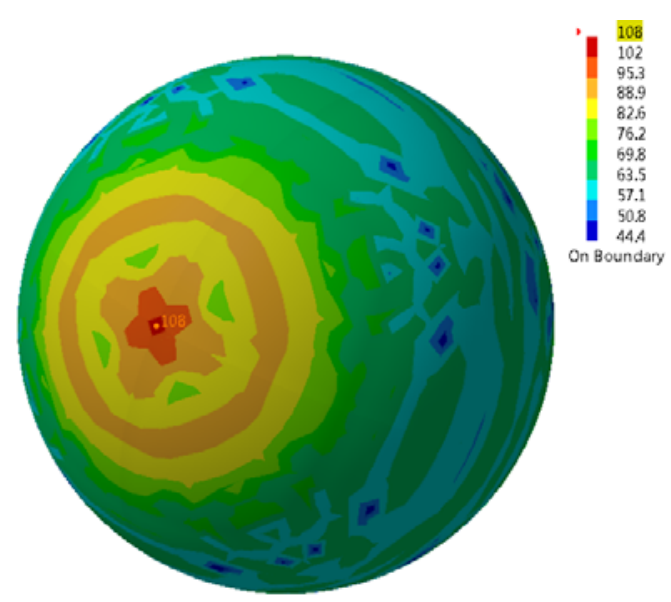

(d) SPLs of wheel ratio 0.4

Fig. 7 SPLs of different wheel ratio modification tasks 
Table 3 Modification tasks of wheel ratio and corresponding maximum SPLs

\begin{tabular}{|c|c|c|c|c|}
\hline Task & 1 & 2 & 3 & 4 \\
\hline Wheel ratio & 0.25 & 0.30 & 0.35 & 0.40 \\
\hline Thrust (N) & 3123.9 & 3110.4 & 3013.3 & 2880.3 \\
\hline SPLs (dB) & 82.9 & 85.8 & 101 & 108 \\
\hline
\end{tabular}

Relatively high wheel ratio generates high noise (Tab. 3). In most wheel ratio, when the ratio is 0.25, its acoustic noise is the lowest among the four cases (Fig.6; Tab. 3), so its overall noise is the lowest, with the corresponding maximum SPL being $82.9 \mathrm{~dB}$. Thus, the change of wheel ratio has a big impact on the aerodynamic and acoustic performance of turbofan engine fan.

\section{Summary}

This paper aims to predict and reduce the acoustic noise of turbofan engine fan. The acoustic characteristics of turbofan engine fan were investigated by using the thin-body BEM/FW-H method. And then the effects of two main design parameters, including blade angle and wheel ratio were discussed. These design parameters have obvious influence on the acoustic performance of turbofan engine fan. Some meaningful conclusions are drawn on the noise reduction. By modifying the two main design parameters, the SPL decrease by $10.3 \mathrm{~dB}$ compared with the original model. These results demonstrate the validity of design parameters modification of turbofan engine fan in acoustic noise reduction. In the following research, the noise reduction effect of other design parameters can be investigated.

\section{References}

[1]. D.K.M. Argüelles, O.J.M. Fernández, M.E. Blanco, et al. Numerical prediction of tonal noise generation in an inlet vaned low-speed axial fan using a hybrid aeroacoustic approach. Journal of Mechanical Engineering Science. Vol. 223 (2009) No. 9, p. 2081-2098.

[2]. Y. Wang, G.H. Xu, Q.J. Zhao. Numerical method for predicting rotor aerodynamic noise based on unstructured-grid CFD technology. Acta Aerodynamica Sinica. Vol. 29 (2011) No. 5, p. 559-566.

[3]. Tyler, J. M., and Sofrin, T.G., Axial Flow Compressor Noise Studies[J]. SAE transactions, 1962, 70:309-332.

[4]. Yuntao Cao. Fast Analysis and Optimization of Performance of Heavy-Duty Vehicle Engine Cooling Fan [D]. Changchun: Jilin University, 2009, 43-42

[5]. Akaike S, Kikuyama K, Kitada M, et al. Study of rotational noise reduction for axial flow fan[J]. JSME International Journal, Series B, 1996, 39(3): 590-596

[6]. G. Srinivasan, W. McCroskey. Navier-Stokes calculations of hovering rotor flowfields. Journal of Aircraft. Vol. 25 (1988) No. 10, p. 865-874.

[7]. K.S. Brentner, F. Farassat. Analytical Comparison of the Acoustic Analogy and Kirchhoff Formulation for Moving Surfaces. AIAA Journal. Vol. 36 (1998) No. 8, p. 1379-1386.

[8]. D. Fedala, S. Kouidri, R. Rey. Numerical study of time domain analogy applied to noise prediction from rotating blades. Journal of Sound and Vibration. Vol. 321 (2009) No. 3-5, p. 662-679.

[9]. M. Carley. The Sound Field of a Rotor in a Stationary Duct. Journal of Sound and Vibration. Vol. 259 (2003) No. 5, p. 1067-1079.

[10]. B.B. Hu, H. OuYang, Y.D. Wu, et al. Numerical prediction of the interaction noise radiated from an axial fan. Applied Acoustics. Vol. 74 (2013) No. 4, p. 544-552. 